\title{
Production of a Beam of Tensor-Polarized Deuterons Using a Carbon Target
}

\author{
H. Seyfarth, ${ }^{1, *}$ R. Engels, ${ }^{1}$ F. Rathmann, ${ }^{1}$ H. Ströher, ${ }^{1}$ V. Baryshevsky, ${ }^{2}$ A. Rouba, ${ }^{2}$ C. Düweke,${ }^{3, \dagger}$ R. Emmerich, ${ }^{3, \dagger}$ \\ A. Imig, ${ }^{3,8}$ K. Grigoryev, ${ }^{1,4}$ M. Mikirtychiants, ${ }^{1,4}$ and A. Vasilyev ${ }^{4}$ \\ ${ }^{1}$ Institut für Kernphysik, Jülich Center for Hadron Physics, Forschungszentrum Jülich, Leo-Brandt-Straße 1, D-52425 Jülich, \\ Germany \\ ${ }^{2}$ Research Institute for Nuclear Problems, Bobruiskaya Straße 11, 220050 Minsk, Belarus \\ ${ }^{3}$ Institut für Kernphysik, Universität zu Köln, Zülpicher Straße 77, D-50937 Köln, Germany \\ ${ }^{4}$ Petersburg Nuclear Physics Institute, 188300 Gatchina, Russia
}

(Received 26 November 2009; published 3 June 2010)

\begin{abstract}
An initially unpolarized beam of deuterons is found to acquire tensor polarization after traversing a foil of spin-zero target nuclei. The effect, called nuclear spin dichroism, has been predicted theoretically, albeit resulting in small values of $p_{z z}$ of the order of 0.01 for energies around $10 \mathrm{MeV}$. The experiment was carried out at the Köln tandem accelerator using carbon targets bombarded by deuterons. The observed polarization is as large as $p_{z z}=-0.28 \pm 0.03$ for a beam of $14.8 \mathrm{MeV}$ and a $129 \mathrm{mg} / \mathrm{cm}^{2} \operatorname{target}$. The results allow one to produce tensor-polarized deuterons with $p_{z z}$ around -0.30 (or +0.25 ) from an initially unpolarized beam using a carbon target of appropriate thickness.
\end{abstract}

DOI: 10.1103/PhysRevLett.104.222501

Nuclear spin dichroism leads to the appearance of tensor polarization in an initially unpolarized, forwardtransmitted beam of (spin 1) deuterons behind a target composed of spin-zero nuclei, like carbon [1]. The direction of primary and transmitted beam defines the quantization axis, and because of azimuthal symmetry, the beam behind the target is described by the tensor polarization

$$
p_{z z}(\rho d)=n_{+1}(\rho d)+n_{-1}(\rho d)-2 n_{0}(\rho d),
$$

where $\rho$ is the number density of nuclei in the target (per unit of volume) and $d$ its thickness (in units of length). The $n_{m}$ denote the normalized occupation numbers of deuterons in the magnetic substates $m=+1,-1$, and 0 . The interaction of deuterons of energy $E$ with spin-zero target nuclei is characterized by the total cross sections $\sigma_{+1}(E)$ and $\sigma_{0}(E)$. Using the initial occupation numbers in the beam, $n_{+1}^{\text {in }}=n_{-1}^{\text {in }}=n_{0}^{\text {in }}=\frac{1}{3}$, the tensor polarization behind the target reads

$$
p_{z z}(\rho d)=\frac{2}{3}\left[\exp \left(-\rho \Sigma_{ \pm 1}\right)-\exp \left(-\rho \Sigma_{0}\right)\right],
$$

where $\Sigma_{m}=\int_{0}^{d} \sigma_{m}[E(x)] d x$, and $E(x)$ is the deuteron energy at a penetration depth $x$ into the target. When $\rho \Sigma_{m} \ll 1$, Eq. (2) reduces to

$$
p_{z z}(\rho d)=\frac{2}{3} \rho \int_{0}^{d}\left\{\sigma_{0}[E(x)]-\sigma_{ \pm 1}[E(x)]\right\} d x .
$$

A nonzero cross-section difference is produced, for instance, by the nonspherical shape of the deuteron ground-state wave function.

Recently, the deuteron-carbon interaction at deuteron energies of 5 to $20 \mathrm{MeV}$ has been investigated theoretically [1]. The optical theorem relates the cross-section difference in Eq. (3) to the imaginary part of the coherent forward scattering amplitudes,
PACS numbers: 13.88.+e, 24.70.+s, 25.45.De, 29.27.Hj

$$
\sigma_{0}(E)-\sigma_{ \pm 1}(E)=\frac{4 \pi}{k} \Im\left[f_{0}^{0}(E)-f_{ \pm 1}^{0}(E)\right],
$$

where $k$ is the deuteron wave number in the deuteronnucleus potential $V_{d}$. The difference $f_{0}^{0}(E)-f_{ \pm 1}^{0}(E)$ is calculated in Ref. [1] in eikonal approximation for $E \gg$ $V_{d}$ with a correction term for $E \leq V_{d}$. Neglecting changes in the deuteron wave function in the interaction process, the main contributions to the difference are due to the nuclear interaction of neutron and proton in the deuteron with target nucleons $(\mathrm{NN})$, and the interference in the nuclear and Coulomb interaction of the nucleons (NNC). The cross-section difference $\sigma_{0}(E)-\sigma_{ \pm 1}(E)$, caused by the $\mathrm{NN}$ interaction, is positive, slightly decreasing from $2.5 \mathrm{~b}$ at $5 \mathrm{MeV}$ to $2.0 \mathrm{~b}$ at $20 \mathrm{MeV}$. The correction term yields a small positive contribution. The NNC interaction, however, produces a sign change from negative values below $15 \mathrm{MeV}$ to positive values above. Superpositioning of all three contributions yields a small positive total crosssection difference around 5.0 to $5.5 \mathrm{MeV}$, a negative one between 5.5 and $11 \mathrm{MeV}$ with a minimum of about $-6 \mathrm{~b}$ at $7.5 \mathrm{MeV}$, and again positive values above $11 \mathrm{MeV}$, approaching $+3 \mathrm{~b}$ around $20 \mathrm{MeV}$. For relativistic energies, 2 orders of magnitude smaller positive values of $p_{z z}$ had been predicted [2], recently confirmed by a measurement with $5.5 \mathrm{GeV} / c$ deuterons interacting with carbon targets [3].

When Eq. (3) is integrated over the range $E_{\text {in }}=E(x=$ $0)$ to $E_{\text {out }}=E(x=d)$, deuterons decelerated from 20 to $11 \mathrm{MeV}$ (or from 11 to $5.5 \mathrm{MeV}$ ) in a carbon target of areal density 180 (or 70) $\mathrm{mg} / \mathrm{cm}^{2}$ are expected to acquire $p_{z z}=$ +0.014 (or $p_{z z}=-0.0035$ ) [1]. These predictions are to be compared with the experimental data obtained in this work.

The measurements, presented here, were performed in two runs, in 2003 and 2006, with unpolarized deuteron 
beams from the HVEC FN Van de Graaff tandem accelerator of the Institut für Kernphysik of the Universität zu Köln. The tensor polarization produced in seven carbon targets of different thickness was determined using a polarimeter [4] based on the $\vec{d}+{ }^{3} \mathrm{He} \rightarrow p+{ }^{4} \mathrm{He}$ reaction. The polarimeter setup is depicted in Fig. 1. Deuterons emitted from the target into the polarimeter cell were confined by diaphragms to polar angles $\theta \leq 0.5^{\circ}$. Protons from the polarimeter reaction, emitted at $\theta=(24.5 \pm 2.9)^{\circ}$, were detected by four side detectors positioned at azimuthal angles $\varphi=0^{\circ}, 90^{\circ}, 180^{\circ}$, and $270^{\circ}$ [labeled $L$ (left), $U$ (up), $R$ (right), and $D$ (down), respectively, as seen in beam direction], and by a forward detector (labeled $F$ ) for protons emitted at $\theta \leq 2.6^{\circ}$. The ${ }^{3} \mathrm{He}$ cell and the diaphragms, electrically insulated from each other and from the other components, were used to monitor the beam currents. The primary beam for all targets was tuned to deliver currents of $\approx 7 \mathrm{nA}$ to the polarimeter cell.

In steps of $0.1 \mathrm{MeV}$, a set of primary beam energies $E_{\text {in }}$ was used for each target, chosen such that the deuteron energies $E_{c}$ in the ${ }^{3} \mathrm{He}$ gas cell of the polarimeter ranged from 5 to $8 \mathrm{MeV}$ (see Table I). At these energies, the tensor analyzing powers of the polarimeter reaction are large and vary smoothly, $A_{z z}\left(0^{\circ}\right)=-1.75$ to -1.65 , and $A_{z z}\left(24.5^{\circ}\right)=-0.84$ to -0.97 [4]. The appropriate $E_{\text {in }}$ were calculated using the Bethe-Bloch equation, taking into account the energy loss in the carbon targets, the Havar window, and the mean penetration depth of $13 \mathrm{~mm}$ in the ${ }^{3} \mathrm{He}$ gas.

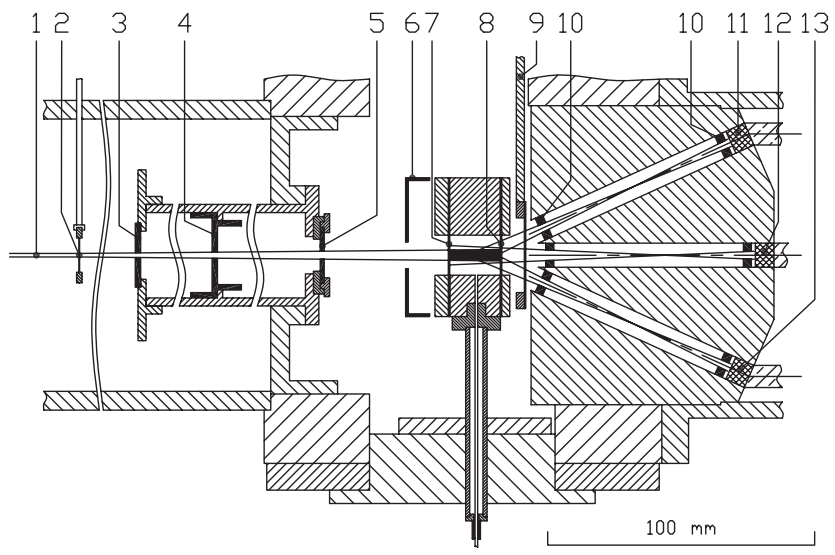

FIG. 1. View from the top along the horizontal midplane of the polarimeter: (1) unpolarized beam $(\phi=1.5 \mathrm{~mm})$; (2) target foil (s) in an aluminum frame; (3-5) diaphragms $D_{1}(\phi=2.0 \mathrm{~mm})$, $D_{2}(\phi=2.5 \mathrm{~mm}), D_{3}(\phi=3.0 \mathrm{~mm})$; (6) electron-backbending electrode; (7) $6.5 \mu$ Havar entrance window of the polarimeter cell (filled with ${ }^{3} \mathrm{He}$ gas of $3 \mathrm{bar}, l=18 \mathrm{~mm}, \phi=14 \mathrm{~mm}$ ); (8) tantalum exit window of $100 \mu \mathrm{m}$ thickness to stop the residual deuterons during the carbon runs; (9) additional $300 \mu \mathrm{m}$ tantalum foil on a sliding ladder inserted during beam tuning without target; (10) tantalum apertures; (11-13) NaI(Tl) detectors with light guides to the Philips XP1911 photomultipliers. The distances from the target were 132, 187, and $251 \mathrm{~mm}$ for $D_{1}, D_{2}$, and $D_{3}$, and $299 \mathrm{~mm}$ for the Havar window.
The target foils were cut from rolled sheets of expanded graphite [5]. The target thicknesses and their uncertainties were determined from about 100 circular samples. The nominal $99 \%$ purity of carbon was confirmed by analyses employing standard techniques [6]. Except for C36 and C58 (see Table I), all targets consisted of stacks of at least two foils of available thickness. The measurement with a gold foil was performed to obtain reference data with a target simulating multiple small-angle Coulomb scattering in the carbon targets without, however, production of polarization.

In all spectra, obtained with the five detectors, the proton peak was well separated from the background, caused by $\gamma$ radiation and scattered protons. The yields in the proton peaks were extracted using fit functions, composed of three Gaussians to account for the asymmetric shape of the peaks, together with an exponential and a modified error function to fit the background. The number of counts in the detectors $(i=L, R, U, D$, and $F$ ) is expressed by

$$
N_{i}\left(E_{c}\right)=C_{i} \sigma_{0}\left(E_{c}, \theta_{i}\right)\left[1+\frac{1}{2} p_{z z}\left(E_{c}\right) A_{z z}\left(E_{c}, \theta_{i}\right)\right],
$$

where the $C_{i}$ depend on the deuteron current in the polarimeter cell, the gas density in the cell, the detector acceptances, and the efficiencies. The $\sigma_{0}\left(E_{c}, \theta_{i}\right)$ are the unpolarized differential cross section of the polarimeter reaction with proton emission at $\theta_{i=L, R, U, D}=24.5^{\circ}$ or $\theta_{i=F}=0^{\circ}$. In order to compensate the dependence of the count rates in the detectors on beam focusing, typically observed as intensity fluctuations of $\sim 5 \%$, the proton-peak ratios

$$
r\left(E_{c}\right)=\frac{N_{L}\left(E_{c}\right)+N_{U}\left(E_{c}\right)+N_{R}\left(E_{c}\right)+N_{D}\left(E_{c}\right)}{N_{F}\left(E_{c}\right)}
$$

were used in the data analysis.

In good approximation, the unpolarized cross sections [7] yield a linear dependence of the proton-peak ratios $r\left(E_{c}\right)$ in the range $E_{c}=5$ to $8 \mathrm{MeV}$. The parametrization $r\left(E_{c}\right)=(1.58 \pm 0.05)-(0.119 \pm 0.006) E_{c}(\mathrm{MeV})$ agrees well with the one of the Au5 target, $r_{\mathrm{fit}}^{\mathrm{Au} 5}\left(E_{c}\right)=(1.65 \pm$ $0.01)-(0.116 \pm 0.002) E_{c}$. This implies that only a negligibly small polarization is produced in the Au5 target. For that reason, Au5 is used here as a reference target. Figure 2 shows the deviation of ratios $r^{\mathrm{Cx}}\left(E_{c}\right)$ and linear fits $r_{\mathrm{fit}}^{\mathrm{Cx}}\left(E_{c}\right)$ from the reference target $r_{\text {fit }}^{\text {Aus }}$. Large deviations and a strong dependence on $E_{c}$ (and accordingly on $E_{\text {in }}$ ) were observed, indicating a significant value of $p_{z z}$ in the forward-transmitted beam behind the carbon target. The effect is most pronounced for the $\mathrm{C} 129$ target, for which the measured ratios $r^{\mathrm{C} 129}\left(E_{c}\right)$ were $0.808 \pm 0.006$ and $0.665 \pm 0.007$ at $E_{c}=5.56$ and $7.33 \mathrm{MeV}$. The corresponding values for the Au5 reference target yielded $r^{\mathrm{Au} 5}\left(E_{c}\right)=0.911 \pm 0.002, \quad$ and $\quad 0.705 \pm 0.002$, respectively.

Based on Eqs. (5) and (6), and the measured parametrizations of the peak ratio for the Au5 and the carbon targets, 
TABLE I. Target material $M$ and thickness $d_{t}$, target label, run (I, 2003; II, 2006), minimal and maximal primary deuteron beam energies $E_{\text {in }}$, and minimal and maximal mean deuteron energies $E_{c}$ in the ${ }^{3} \mathrm{He}$ polarimeter cell.

\begin{tabular}{cccccccc}
\hline \hline & & \multicolumn{2}{c}{$E_{\text {in }}[\mathrm{MeV}]$} & \multicolumn{2}{c}{$E_{c}[\mathrm{MeV}]$} \\
$M$ & $d_{t}\left[\mathrm{mg} / \mathrm{cm}^{2}\right]$ & Label & Run & Min & Max & Min \\
\hline $\mathrm{Au}$ & $5.0 \pm 0.3$ & Au5 & II & 6.20 & 7.90 & 5.56 & 7.36 \\
$\mathrm{C}$ & $35.90 \pm 0.19$ & C36 & II & 9.50 & 10.50 & 6.06 & 7.41 \\
$\mathrm{C}$ & $57.69 \pm 0.32$ & C58 & I & 10.80 & 12.20 & 5.73 & 7.83 \\
$\mathrm{C}$ & $93.59 \pm 0.37$ & C94 & II & 13.00 & 14.00 & 5.72 & 7.46 \\
$\mathrm{C}$ & $129.49 \pm 0.42$ & C129 & II & 14.80 & 15.90 & 5.41 & 7.55 \\
$\mathrm{C}$ & $152.63 \pm 0.75$ & C153 & I & 16.20 & 16.70 & 5.94 & 6.97 \\
$\mathrm{C}$ & $165.39 \pm 0.46$ & C165 & II & 16.70 & 17.50 & 5.70 & 7.39 \\
$\mathrm{C}$ & $187.93 \pm 0.74$ & C188 & I & 17.50 & 18.70 & 5.11 & 7.78 \\
\hline \hline
\end{tabular}

$r_{\text {fit }}^{\mathrm{Au} 5}$ and $r_{\mathrm{fit}}^{\mathrm{Cx}}\left(E_{c}\right)$, the following expression for the tensor polarization of the beam behind the target is obtained,

$$
p_{z z}^{\mathrm{Cx}}\left(E_{c}\right)=\frac{2\left[r_{\mathrm{fit}}^{\mathrm{Au} 5}\left(E_{c}\right)-r_{\mathrm{fit}}^{\mathrm{Cx}}\left(E_{c}\right)\right]}{r_{\mathrm{fit}}^{\mathrm{Cx}}\left(E_{c}\right) A_{z z}\left(E_{c}, 0^{\circ}\right)-r_{\mathrm{fit}}^{\mathrm{Au} 5}\left(E_{c}\right) A_{z z}\left(E_{c}, 24.5^{\circ}\right)} .
$$

Third-order polynomial fits to the data at $\theta=0^{\circ}[4,8,9]$ and $\theta=24.5^{\circ}[4,7,9]$ yield the tensor analyzing powers $A_{z z}$ as function of $E_{c}$.

In Fig. 3, we show the resulting tensor polarization for the different targets used in the measurement. It is obvious from Eq. (3) that, for two targets of different thickness but identical $E_{\text {out }}$, the difference in $p_{z z}$ has to be attributed to the additional energy loss in the thicker target. Therefore, the seven sets of $p_{z z}^{\mathrm{Cx}}$ are plotted as a function of the primary beam energy $E_{\text {in }}$. The error bands take into account the uncertainties of the target thickness, which alter

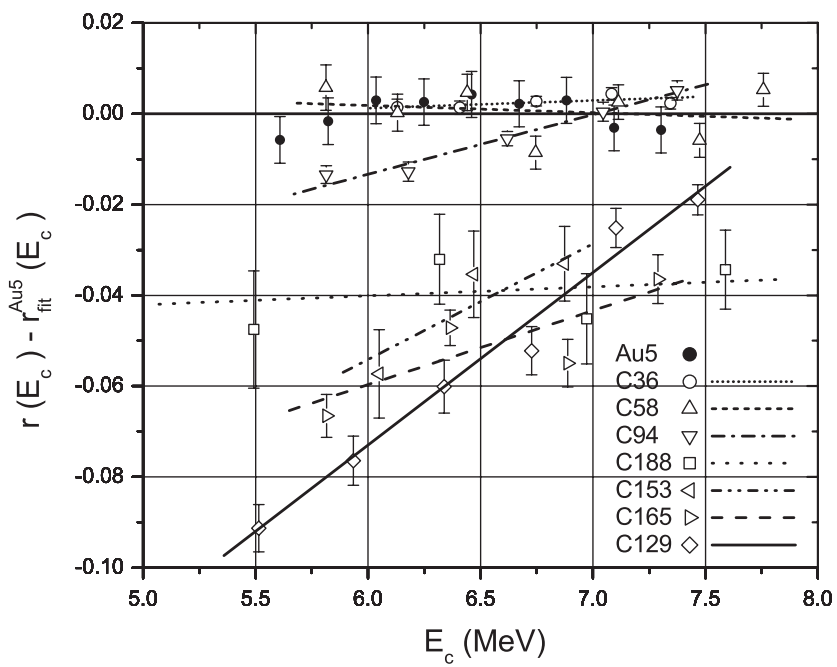

FIG. 2. Difference between fits and measured ratios $r\left(E_{c}\right)$ and the fit to the Au5 data. To ease readability, data points were combined. The fit lines cover the energy ranges during the measurement (Table I). The given errors include the statistical errors of the measured ratios and the sample standard deviation [13] of the fit to the Au5 ratios.
$E_{c}$ and hence modify $p_{z z}$ [see Eq. (7)]. By quadratic superposition of the sample standard deviations for the Au5 and the carbon target, the fitted errors of $r\left(E_{c}\right)$ were added to those resulting from the uncertainties of the target thicknesses.

For deceleration from 14.8 to $14.0 \mathrm{MeV}$, the observed values of $p_{z z}=-0.28 \pm 0.03$ for the C129 target at $E_{\text {in }}=$ $14.8 \mathrm{MeV}$, and $p_{z z} \sim 0$ for the C94 target at $E_{\text {in }}=$ $14.0 \mathrm{MeV}$, imply $\sigma_{0}\left(E_{\text {in }}\right)-\sigma_{ \pm 1}\left(E_{\text {in }}\right)<0$. The decrease of $\left|p_{z z}\right|$ above $14.8 \mathrm{MeV}$ indicates that $\sigma_{0}\left(E_{\text {in }}\right)-$ $\sigma_{ \pm 1}\left(E_{\text {in }}\right)>0$. For the cross-section difference, such a sign change has been predicted recently in Ref. [1], although the calculation favors a smaller energy around $11 \mathrm{MeV}$. In addition, the calculation underestimates both the magnitude and the rapid variation of $\sigma_{0}\left(E_{\mathrm{in}}\right)-$ $\sigma_{ \pm 1}\left(E_{\mathrm{in}}\right)$. The gross features of Fig. 3 are described by $\sigma_{0}-\sigma_{ \pm 1} \sim-550 \mathrm{~b}$ for deceleration from 14.8 to $14.0 \mathrm{MeV}$, and $\sim+300 \mathrm{~b}$ from 15.8 to $14.8 \mathrm{MeV}$, in carbon targets of thickness $d=0.15 \mathrm{~mm} \quad\left(d_{t}=\right.$ $\left.15 \mathrm{mg} / \mathrm{cm}^{2}\right)$ and $0.23 \mathrm{~mm}\left(23 \mathrm{mg} / \mathrm{cm}^{2}\right)$, respectively. The product $\left(\sigma_{0}-\sigma_{ \pm 1}\right) d$ corresponds to about

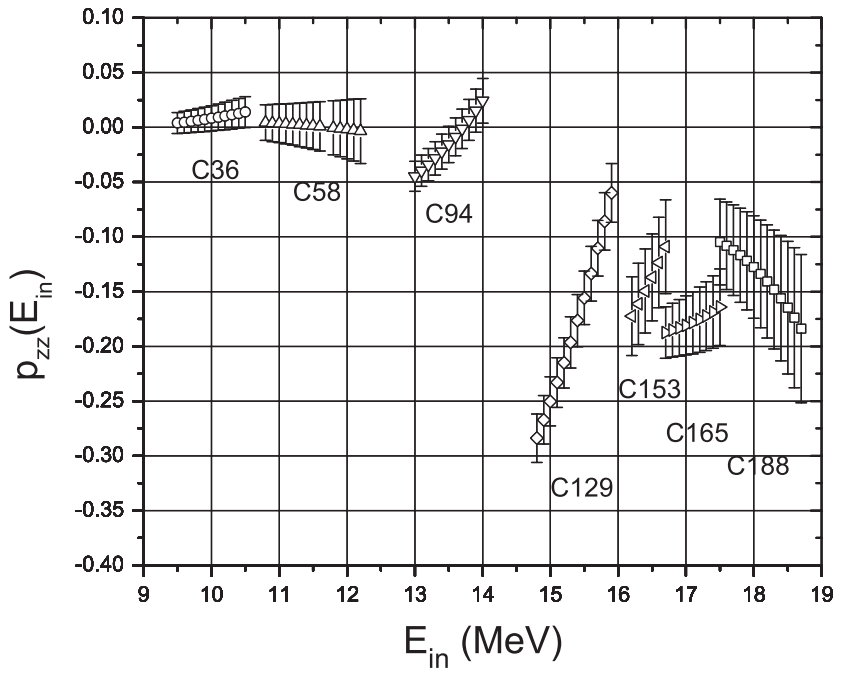

FIG. 3. Deuteron tensor polarizations $p_{z z}$ produced in the seven carbon targets as a function of beam energy $E_{\text {in }}$. 


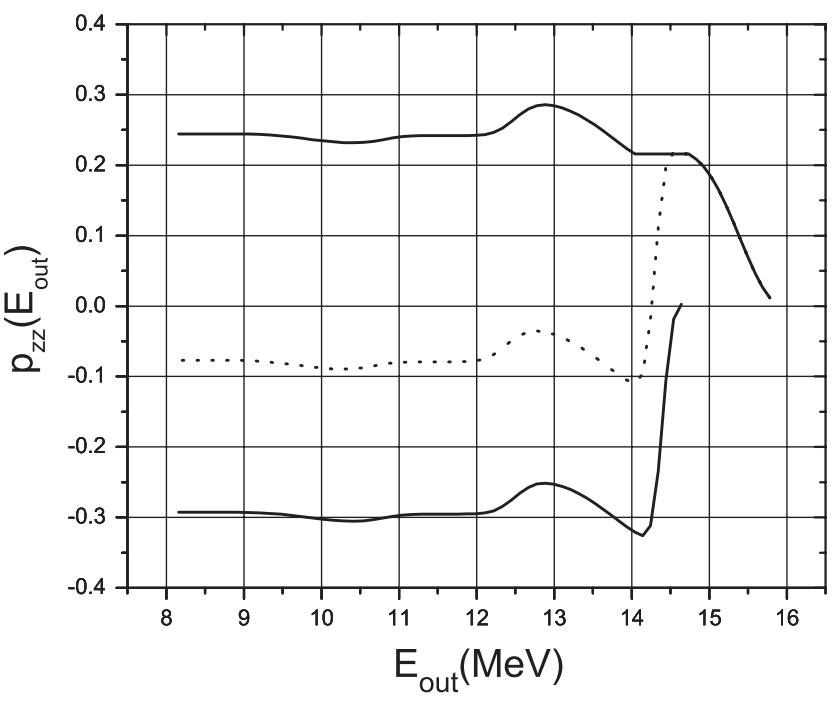

FIG. 4. Tensor polarization $p_{z z}$ produced by graphite targets from unpolarized deuteron beams as a function of the energy $E_{\text {out }}$ behind the target. The lower solid line is for $E_{\text {in }}=$ 14.8 MeV. Both upper lines are for $E_{\text {in }}=15.8 \mathrm{MeV}$ : the solid line is for a target with a different sandwiched material suppressing the negative polarization by carbon around $14.4 \mathrm{MeV}$; the dotted line below $E_{\text {out }}=14.8 \mathrm{MeV}$ is obtained for a pure graphite target.

$-8.5 \mathrm{bcm}$ for deceleration from 14.8 to $14.0 \mathrm{MeV}$, and to about $+6.9 \mathrm{bcm}$ for 15.8 to $14.8 \mathrm{MeV}$.

In a more realistic way, the cross-section differences are described by energy-dependent functions, like Gaussian distributions. The assumption is made that in each of the distributions either $\sigma_{0}(E)$ or $\sigma_{ \pm 1}(E)$ is equal to zero. The two distributions with central energies $E_{0}=14.4$ and 15.4 MeV and additional weaker ones allow one to describe $p_{z z}\left(E_{\text {in }}\right)$ of Fig. 3.

The observed cross-section differences of -550 and $+300 \mathrm{~b}$ are about 2 orders of magnitude larger than the calculated values [1]. Thus, in order to explain the measured energy dependence of $p_{z z}$, additional effects in the deuteron-carbon interaction have to be invoked. These may be spin-orbit coupling, polarization of the deuteron in the Coulomb field, or a change of the deuteron wave function by the interaction as discussed, e.g., in Ref. [10]. It should be mentioned that the excitation energies, available at the energies $E_{0}$ in the compound system $d+{ }^{12} \mathrm{C}$, are in surprisingly good agreement with those of known states in the giant resonance region of ${ }^{14} \mathrm{~N}$ [11].

The present data indicate the possibility to produce tensor-polarized deuterons by passing an initially unpolarized beam through targets made from graphite. The crosssection distributions with $E_{0}=15.4,14.4,13.8,12.5,10.8$, and $9.8 \mathrm{MeV}$ allow one to predict the expected $p_{z z}$ for primary beam energies of 14.8 and $15.8 \mathrm{MeV}$, as shown in Fig. 4. An unpolarized primary deuteron beam of $E_{\text {in }}=$ 14.8 $\mathrm{MeV}$ is expected to leave a carbon target with $p_{z z}$ around -0.30 and $E_{\text {out }}$ depending on the target thickness. For $E_{\text {in }}=15.8 \mathrm{MeV}$ a thin carbon target of $20 \mathrm{mg} / \mathrm{cm}^{2}$ only with $E_{\text {out }}=14.8 \mathrm{MeV}$ would yield $p_{z z}=+0.21$. Smaller $E_{\text {out }}$ and even slightly larger tensor polarizations would be achieved, when the deceleration from 14.8 to 14.0 MeV would take place in a target material that does not exhibit a polarizing effect.

In summary, an unpolarized deuteron beam of $14.8 \mathrm{MeV}$ is found to acquire a tensor polarization $p_{z z}=-0.28 \pm$ 0.03 after traversing a foil of graphite of $129 \mathrm{mg} / \mathrm{cm}^{2}$. This finding could have a potential impact on proposed searches for deuteron electric dipole moments in storage rings [12], since the present concepts of polarimetry rely on elastic $\vec{d} \mathrm{C}$ scattering.

The authors would like to thank the staff of the Institut für Kernphysik of the Universität zu Köln for providing the facilities for this experiment, and, in particular, Hans Paetz gen. Schieck for his support during the measurements. We would also like to thank Kolya Nikolaev and Siegfried Krewald for useful discussions.

\section{*H.Seyfarth@fz-juelich.de}

†resent address: AREVA NP GmbH, 91058 Erlangen, Germany.

"Present address: TU München, Physics Department, 85748 Garching, Germany.

${ }^{\S}$ Present address: BNL, Upton, NY 11973, USA.

[1] V. Baryshevsky and A. Rouba, Phys. Lett. B 683, 229 (2010).

[2] G. Fäldt, J. Phys. G 6, 1513 (1980).

[3] L.S. Azhgirey et al., Part. Nuclei Lett. 5, 728 (2008) [http://www1.jinr.ru/Pepan_letters/Letters_eng_arh.html].

[4] R. Engels, Diploma thesis, Universität zu Köln, 1997 [available at http://www.ikp.uni-koeln.de/groups/ex/ schieck/arbeiten/engels.diplom.pdf].

[5] Manufactured by immersing natural graphite flakes in a bath of chromic acid, then concentrated sulfuric acid, which forces the crystal lattice planes apart, thus expanding the graphite.

[6] Performed by Zentralabteilung für Chemische Analysen (ZCH) of Forschungszentrum Jülich $\mathrm{GmbH}$.

[7] M. Bittcher et al., Few-Body Syst. 9, 165 (1990).

[8] P. A. Schmelzbach et al., Nucl. Phys. A264, 45 (1976).

[9] S. A. Tonsfeldt, Ph.D. thesis, University of North Carolina, 1983, available from University Microfilms, Ann Arbor, MI, USA.

[10] H. Lenske and G. Baur, Nucl. Phys. A344, 151 (1980).

[11] F. Ajzenberg-Selove, Nucl. Phys. A523, 1 (1991).

[12] B. Lee Roberts, James P. Miller, and Yannis K. Semertzidis, in Lepton Dipole Moments, edited by B. Lee Roberts and William J. Marciano, Advanced Series on Directions in High Energy Physics Vol. 20 (World Scientific, Singapore, 2010).

[13] P. R. Bevington, Data Reduction and Error Analysis for the Physical Sciences (McGraw-Hill, New York, 1969). 